\title{
STREAMWISE DYNAMICS CONTROLLED JET SPREADING, MIXING AND PHYSICAL SOURCE OF THE VORTICES
}

\section{Amalendu Sau}

Department of Mechanical and Aerospace Engineering, Gyeongsang National University, Gyeongnam, Jinju, South Korea.

Robert R. Hwang

Department of System Engineering and Naval Architecture, National Taiwan Ocean University, Keelung, Taiwan., phhwang@gate.sinica.edu.tw

Follow this and additional works at: https://jmstt.ntou.edu.tw/journal

Part of the Engineering Commons

\section{Recommended Citation}

Sau, Amalendu and Hwang, Robert R. (2011) "STREAMWISE DYNAMICS CONTROLLED JET SPREADING, MIXING AND PHYSICAL SOURCE OF THE VORTICES," Journal of Marine Science and Technology. Vol. 19: Iss. 6, Article 4.

DOI: $10.51400 / 2709-6998.2202$

Available at: https://jmstt.ntou.edu.tw/journal/vol19/iss6/4

This Research Article is brought to you for free and open access by Journal of Marine Science and Technology. It has been accepted for inclusion in Journal of Marine Science and Technology by an authorized editor of Journal of Marine Science and Technology. 


\section{STREAMWISE DYNAMICS CONTROLLED JET SPREADING, MIXING AND PHYSICAL SOURCE OF THE VORTICES}

\section{Acknowledgements}

Authors gratefully acknowledge many constructive discussions with Prof. Subhasish Dey and Dr. W. C.

Yang, and financial support from NSC (grant No. 97-2221-E-001-024), Taiwan. 


\section{STREAMWISE DYNAMICS CONTROLLED JET SPREADING, MIXING AND PHYSICAL SOURCE OF THE VORTICES}

\begin{abstract}
Key words: streamwise dynamics, inflow and outflow vortices, axis switching, mass entrainment.

ABSTRACT

Within the framework of the present investigation we perform Direct Numerical Simulations to study the role of streamwise dynamics in the jet spreading and mixing, and provide a clear understanding about the source of these important vortices in two-step rectangular sudden expansion flows. The present setup is designed to generate higher mass entrainment, and the system generated passive forcing provided the necessary impetus for the sustained growth/evolution of the streamwise vertices, which played the key role in the mixing enhancement. On the other hand, by suitably placing two tiny rectangular 'tabs' on the inlet channel walls, the nature of growth and the dynamics of the streamwise extended threedimensional vortical rollers could be effectively controlled. These vortices through their inflow/outflow type dynamics are found to effectively control the downstream jet spreading; and the associated physical processes either lead to quick axis switching of the jet section, or stop axis switching altogether. In addition, an extensive pressure analysis, as presented here, suggests that the transverse pressure gradient skewing is probably the major source of streamwise vorticity generation for the flow.
\end{abstract}

Amalendu Sau* and Robert R. Hwang**

\section{INTRODUCTION}

Jet control is a major concern in many industrial applications in order to enhance mixing and improve efficiency. In this context attention has gradually shifted toward using the dynamics of the system generated streamwise vortices as a tool to suitably control the developed flow. The experimental findings of Breidenthal [3] and Bernal and Roshko [1] display

Paper submitted 02/25/10; revised 01/17/11; accepted 03/04/11. Author for correspondence: Robert R. Hwang (e-mail: phhwang@gate.sinica.edu.tw).

*Department of Mechanical and Aerospace Engineering, Gyeongsang National University, Gyeongnam, Jinju, South Korea.

**Department of System Engineering and Naval Architecture, National Taiwan Ocean University, Keelung, Taiwan. the important role of the streamwise extended vortical structures in the dynamics of turbulent mixing layers. In addition, for a round jet, the predictions by Liepmann and Gharib [11] widely demonstrate the important role of streamwise vortices in the process of enhancing entrainment. Their observations reveal that the streamwise vortices actively control the sustained inward flow of the ambient fluid, which eventually contributes to the continuous lateral spreading of the jet. Notably, in the light of observations made by Liepmann and Gharib [11], a closer look at the experimental findings concerning rectangular jet flows of Zaman [16] reveals, through the inflow type dynamics of streamwise vortices the ambient fluid can be effectively entrained into the jet core, and at the same time the outflow type of dynamics of the streamwise vortices will eject the jet core fluid out to the ambient. Furthermore, with the introduction of tabs at the nozzle exit [16, 17], the entrainment in a setup can be greatly enhanced by virtue of increased strength of the streamwise vortices. However, much of the associated physically important flow details for the class of flows remained virtually un-addressed.

It has now been experimentally verified that $[8,9]$ the small aspect-ratio (non-circular) nozzles have much higher mass entrainment capacity than that of a circular one. With streamwise evolution the initial shape of these jets is known to experience gradual azimuthal distortion $[4,9]$, and at some downstream location the jets switch axes. To this point, a 45 degree rotation of square jets has also been reported [5]. Moreover, the recent studies $[6,12]$ concerning flows through small aspect-ratio rectangular sudden expansion channels exhibit axis-switching characteristics in such confined geometries as well.

On the issues like physical source of generation of the streamwise vortices and their exact role in the jet spreading, notably, the existing literature is still lacking sufficient dependable evidences. It is, however, generally believed that for incompressible flows the lateral skewing of pressure gradient leads to generation of the streamwise vortices [2]. In a recent computational investigation [12] of flows through a channel with a suddenly expanded and contracted part, we attempted to analyze how spanwise pressure gradient induced lateral flow accelerations can control the generation of these technically important (streamwise) vortices. However, there remains 


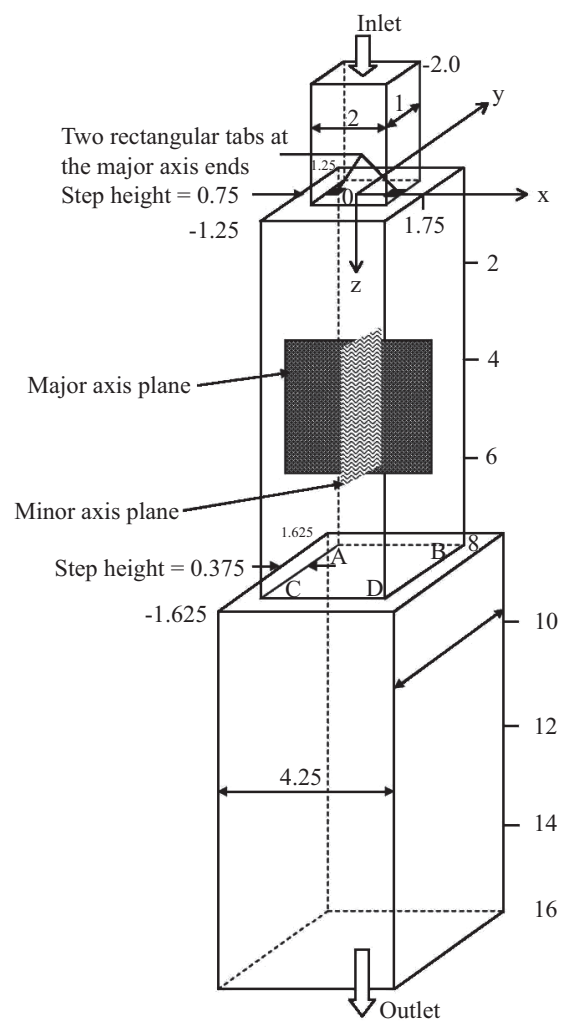

Fig. 1. Schematic of the physical setup.

definite need to further extend the applicability of the proposed pressure analysis by checking its validity for different flow situations. Furthermore, an in-depth analysis of the structural growth of the flow through a two-step rectangular sudden expansion channel, and its proper understanding, particularly focusing on the exact roles of the associated streamwise dynamics, are expected to throw enough light, which may help to considerably improve the existing knowledge of governing physics for much wider class of flows.

\section{THE PHYSICAL PROBLEM}

In the present study incompressible flow simulations are conducted in a 2:1 aspect ratio rectangular channel, which undergoes two consecutive sudden expansions of uniform step heights 0.75 and 0.375 times the minor width of the inlet channel (Fig. 1). The non-dimensional length of the first expansion zone is taken as 8.0, and an equal length is considered for the second expansion zone. Suitable streamwise vortices in the flow field are introduced by means of symmetrically placing two vortex generating 'tabs' (protrusions) on the plane of first expansion, with their bases fixed on the inlet channel wall. The tabs have non-dimensional base width 0.26 and their height leading into the channel has non-dimensional length 0.16 . The area blockage due to each tab on the plane of expansion is approximately $2.1 \%$ of the inlet section area. Computations are performed for Reynolds number, 200, based on the average inlet velocity and the minor inlet channel width.

\section{GOVERNING EQUATIONS AND BOUNDARY CONDITIONS}

The predictions for incompressible flow through the rectangular channel are obtained by numerically solving the following non-dimensional 3D equations of mass and momentum balance:

$$
\begin{gathered}
\frac{\partial u}{\partial x}+\frac{\partial v}{\partial y}+\frac{\partial w}{\partial z}=0 \\
\frac{\partial u}{\partial t}+u \frac{\partial u}{\partial x}+v \frac{\partial u}{\partial y}+w \frac{\partial u}{\partial z}=-\frac{\partial p}{\partial x}+1 / \operatorname{Re}\left[\frac{\partial^{2} u}{\partial x^{2}}+\frac{\partial^{2} u}{\partial y^{2}}+\frac{\partial^{2} u}{\partial z^{2}}\right] \\
\frac{\partial v}{\partial t}+u \frac{\partial v}{\partial x}+v \frac{\partial v}{\partial y}+w \frac{\partial v}{\partial z}=-\frac{\partial p}{\partial y}+1 / \operatorname{Re}\left[\frac{\partial^{2} v}{\partial x^{2}}+\frac{\partial^{2} v}{\partial y^{2}}+\frac{\partial^{2} v}{\partial z^{2}}\right] \\
\frac{\partial w}{\partial t}+u \frac{\partial w}{\partial x}+v \frac{\partial w}{\partial y}+w \frac{\partial w}{\partial z}=-\frac{\partial p}{\partial y}+1 / \operatorname{Re}\left[\frac{\partial^{2} w}{\partial x^{2}}+\frac{\partial^{2} w}{\partial y^{2}}+\frac{\partial^{2} w}{\partial z^{2}}\right]
\end{gathered}
$$

In the equations above, all lengths have been nondimensionalized with respect to the minor channel width $h$ upstream of expansion, the velocities are normalized with respect to the average incoming velocity $W$ at the channel inlet. The Reynolds number of the flow is defined as $R e=W h / v$.

At the inlet section of the channel we use fully developed axial velocity profile with unit mean. On the solid surfaces, the no-slip wall boundary conditions are used. At the downstream end of the expansion channel, the zero gradient outflow conditions were implemented. Computations are performed in a domain with non-dimensional axial lengths $2.125,1.625$ and 16.0 , respectively.

\section{NUMERICAL METHOD}

Using a finite volume method, Eqs. (1)-(4) have been discretized to solve for the velocity and pressure. The convective terms in the momentum equations are discretized by using a third-order accurate upwind (QUICK) scheme, and the viscous terms are discretized using a fourth-order accurate central difference scheme. Extensive description of the schemes and the solution algorithm is presented in [13-15]. An improved version of the Marker-and-Cell (MAC) method was implemented to solve the discretized system of equations. Computations were carried out on a parallelized system of Pentium $1900 \mathrm{MHz}$ processors, in a domain with a spatial resolution of $244 \times 101 \times$ 71 grid points. Notably, in some of our recent works [12-15] we carefully implemented the solution algorithm to study various complex flow phenomena associated with jet flows. 

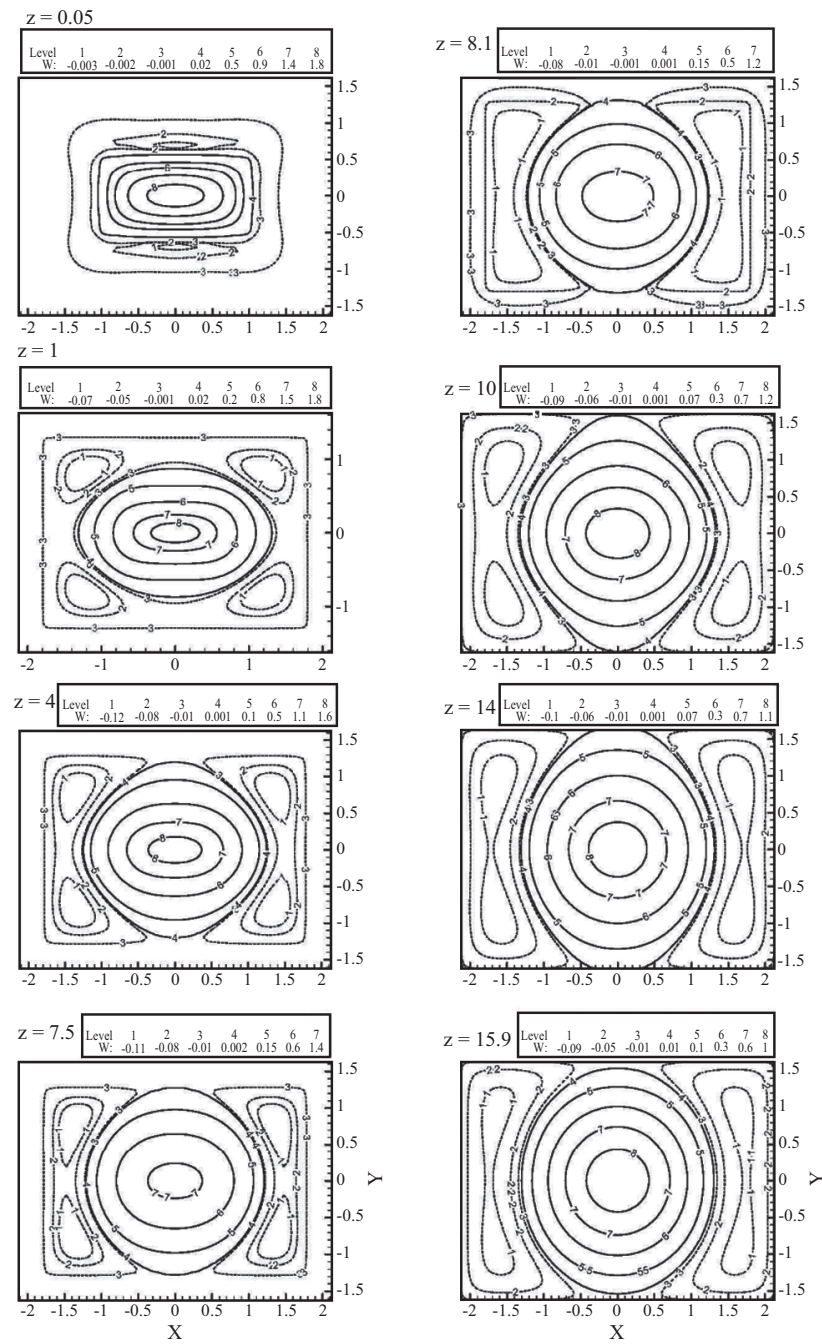

Fig. 2. Contours of the streamwise velocity $(w)$ in absence of tabs; $R e=$ 200.

\section{RESULTS AND DISCUSSION}

Role of streamwise dynamics in controlling various forms of perturbations of the jet is analyzed here. The Reynolds number for the flow is taken as $R e=200$. Fig. 2 displays the structural growth of the jet, through different streamwise stations, in the form of contours of the axial velocity, $w$, in absence of tabs. The dotted contours reveal the structure of the recirculating upstream flow. The sequential distortions of solid contours in Fig. 2, starting immediately behind the plane of first expansion, demonstrate the azimuthal perturbations the jet undergoes during streamwise evolution. While the jet structure is seen to preserve its upstream rectangular shape at $z=$ 0.05 , by the midway $(z=4)$ of the first expansion zone it took a diamond shape because of rapid spreading along the minor axis. The jet in absence of tabs is thereafter seen to gradually become circular, as it approached the end $(z=8)$ of the first expansion zone. Importantly, the presence of the second expansion in the setup is observed to generate additional passive forcing, which initiates further azimuthal jet perturbations.
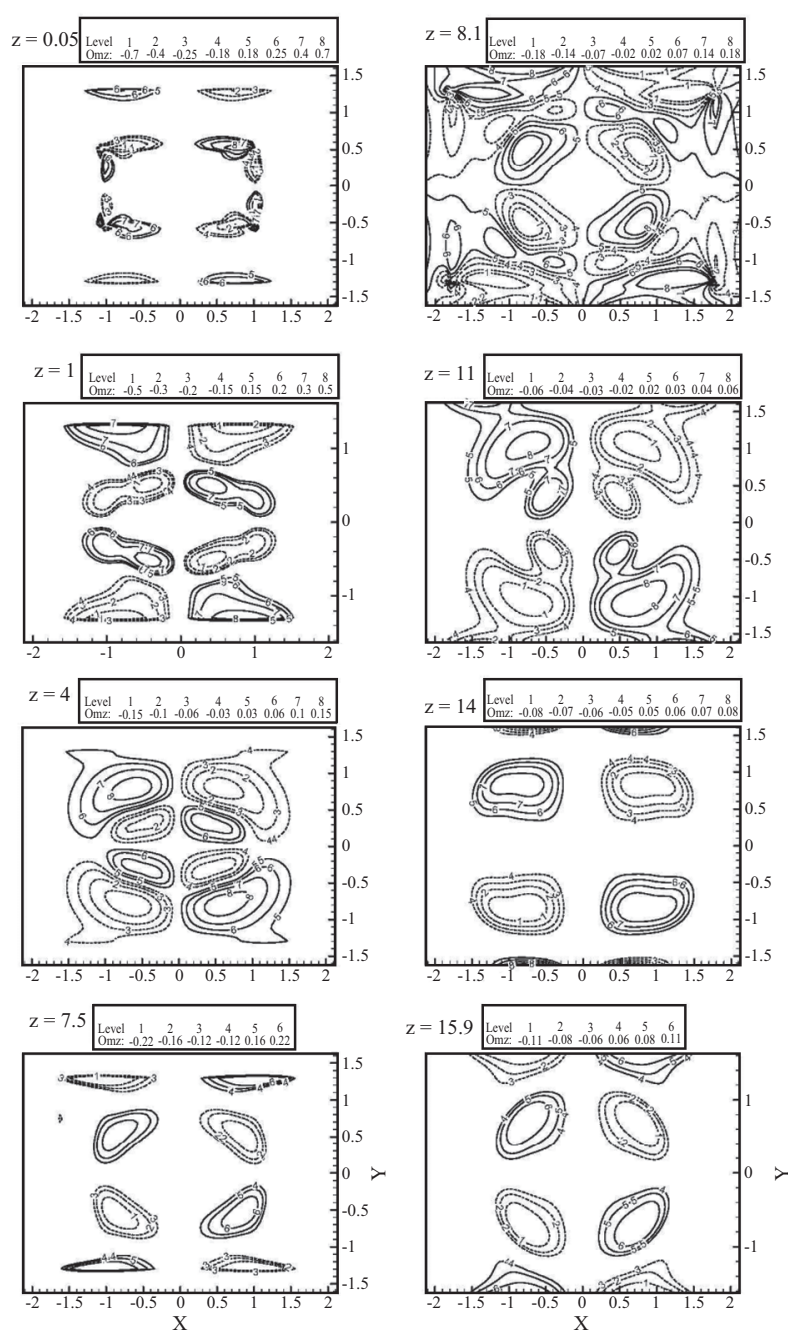

Fig. 3. Contours of streamwise vorticity in absence of tabs; $R \boldsymbol{e}=\mathbf{2 0 0}$.

Fig. 3 shows the streamwise dynamics that the physical system supports. Note that, as soon as flow enters into the first expansion zone, there form four pairs of streamwise vortices, with each inner corner on the expansion plane producing one such pair. However, by $z=1$, we observe two pairs of streamwise vortices to survive, and others get diffused. These vortices are seen to maintain their 'outflow' type nature of dynamics between $1<z<3$; and notably, for $z>4$ there takes place faster growth of passive forcing induced wall vortices at the expense of outflow type core vortices. Such wall vortices are noted to quickly pick up strength and surround/engulf the outflow type core streamwise vortices. Consequently, for $z>6.5$, the outflow type core vortices are seen to get diffused, and the wall vortices (as observed on $z=1$ and $z=4$ ) became the dominant streamwise vortices with their 'inflow' type dynamics. The vorticity contours on $z=11$ reveal one streamwise vortex from each of the four corner pairs (which originated from the plane of second expansion) upon receiving favorable support from the fast growing shear layers along the major widths (e.g. see contours on $z=8.1$ and 11) of the 

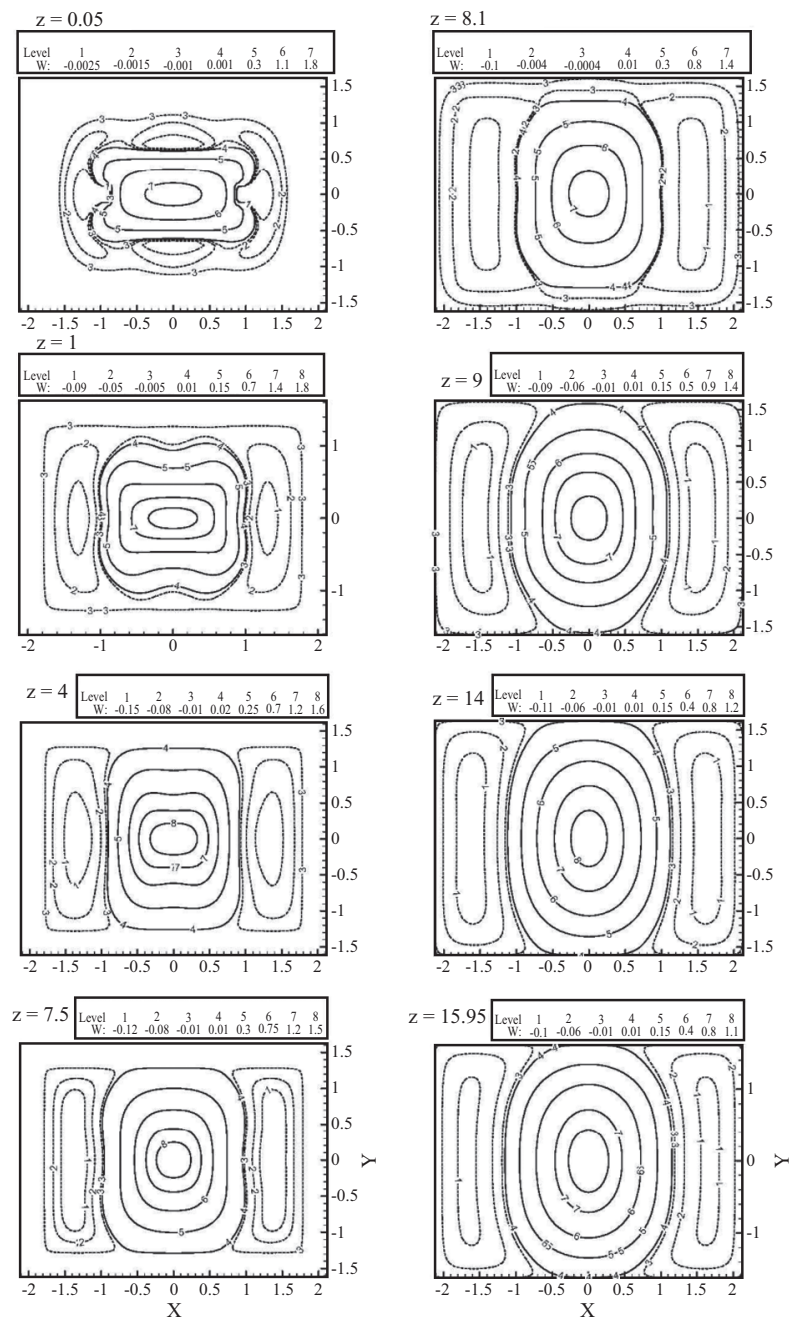

Fig. 4. Contours of the streamwise $(w)$ velocity in presence of two rectangular tabs placed at the major axis ends; $R e=200$.

channel, emerges stronger with streamwise evolution and subsequently joins (near $z=11$ ) the similar natured neighbouring core vortex.

Fig. 4 shows the flow development associated with two rectangular tabs placed symmetrically at the major axis ends (Fig. 1) of the inlet section. Remarkably, by $z=4$ the jet is now seen to assume another rectangular shape, but with reversed axial orientation. At this position the major and the minor axes of the jet have rotated by 90 degrees. Thereafter, such an axis-switched structure of the jet is seen to sustain through the entire downstream flow. Important to note here, the flow recirculation in the setup now occurs mostly along the minor widths. Fig. 5 presents the corresponding streamwise vorticity dynamics. Note that, out of four pairs of streamwise vortices generated from the tabs, the two inner pairs survive until $z=4$, and thereafter they became quickly dissipated; however, the two outer pairs received strong favorable support from the shear induced wall vortices. For $z>4$ these two outer vortex pairs played the leading role as the dominant streamwise vortices in the setup with their inflow type dynamics.
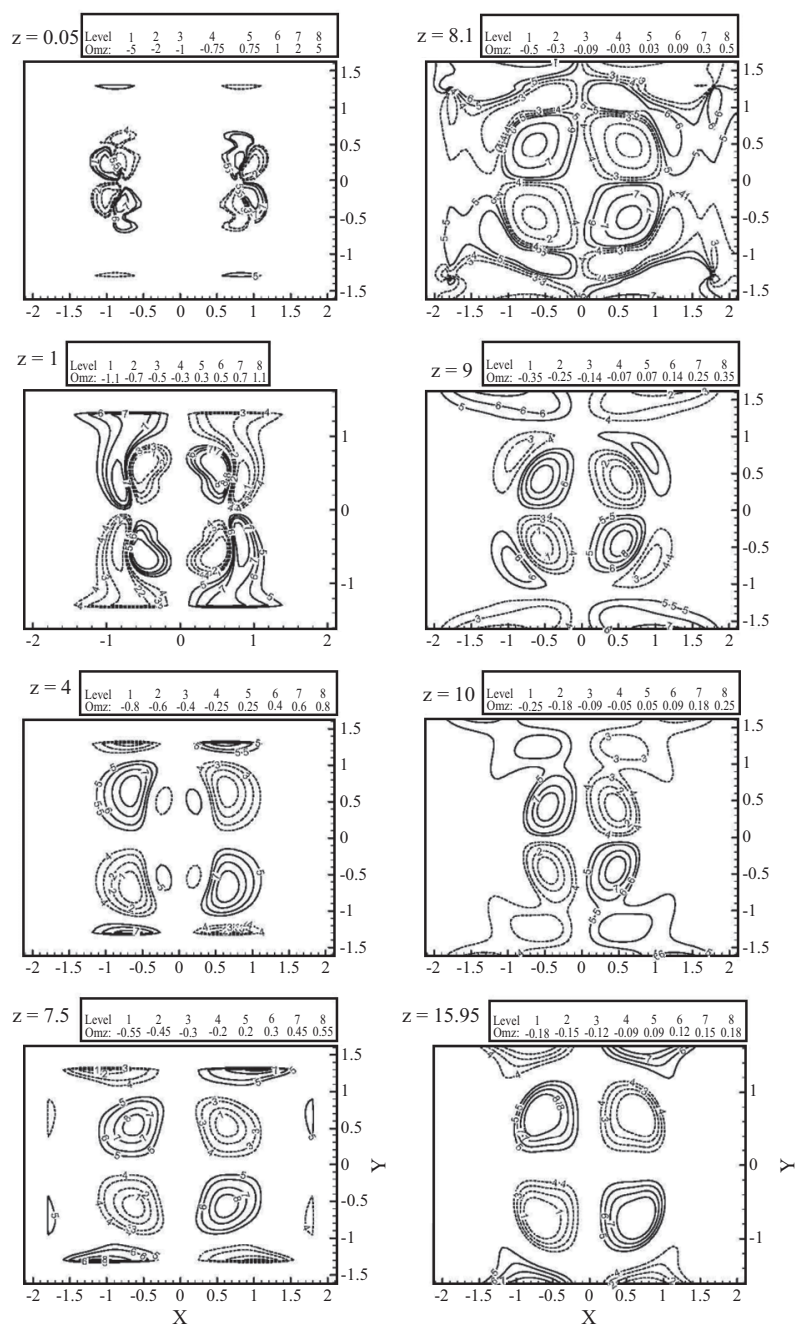

Fig. 5. Contours of streamwise vortices in presence of two rectangular tabs placed at the major axis ends; $\boldsymbol{R e}=\mathbf{2 0 0}$.

With the increased strength, and consequently, due to enhanced induced inward force (particularly due to amalgamation with the like sign vortices originating from the second expansion), the streamwise vortices in this tab configuration (Fig. 5) pushed the jet sufficiently inward from both ends of the major axis, leading to it's quick expansion on the minor axis plane and the eventual axis switching, as shown in Fig. 4.

Fig. 6 shows the detailed spatial growth of the flow in presence of two tabs placed at the minor axis ends of the inlet section. Note that, in contrary to the previous cases, the jet in the present tab orientation continues to expand along the major axis, and the core of the jet exhibits its notable tendency to bifurcate. Fig. 6 further reveals that the azimuthal curvature of the jet on $x=0$ continues to vary steadily over the two expansion zones and the process is rather slow. Notably, in the presence of tabs at the minor axis ends the dominant streamwise vortices (Fig. 7) over the flow domain are noted to evolve in the form of two outflow type pairs. Through outflow type dynamics, the vortices in the present case (Fig. 7) pump the core fluid out to the ambient through the major axis ends and 


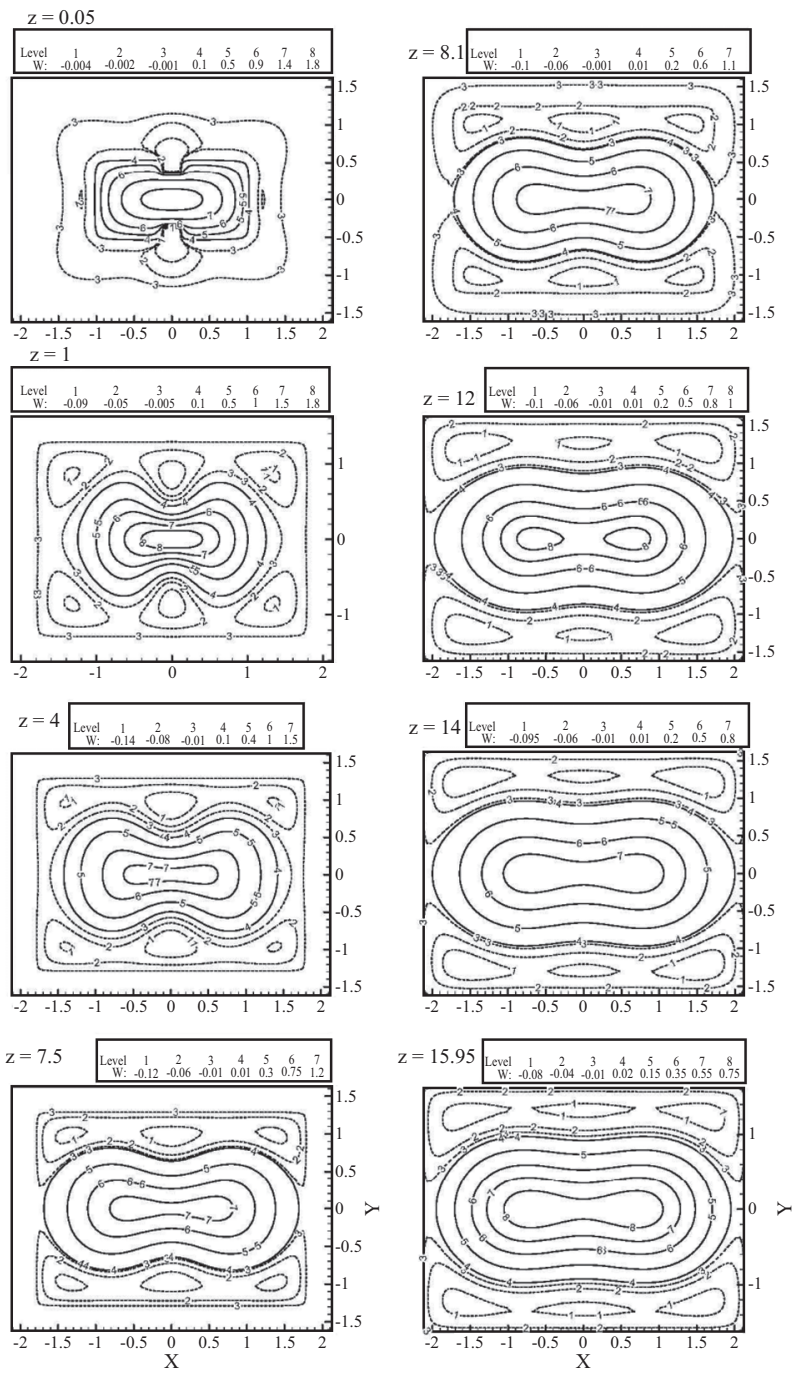

Fig. 6. Contours of the streamwise (w) velocity in presence of two rectangular tabs situated at the minor axis ends; $R e=200$.

they also try to move apart along the major axis, this virtually forced the jet to remain expanded along $\mathrm{x}$-axis. This mechanism is in sharp contrast to the previous two cases (i.e. flow without tabs, as shown in Fig. 3, and flow with tabs at the major axis (Fig. 5) ends), where the dominant streamwise vortices are seen to maintain their inflow type dynamics.

\section{THE SOURCE OF THE STREAMWISE VORTICES}

In Fig. 8 we present the pressure $(p)$ contours on different transverse planes for the flow with two tabs placed at the major axis ends. The corresponding jet evolution pattern and the governing streamwise dynamics have been presented in Figs. 4 and 5, respectively. Firstly, it may be noted that, the depicted iso-contours (Fig. 8) of p reveal formation of several symmetrically placed relative high/low pressure regions at every cross-stream station, which helps to generate non-uniform
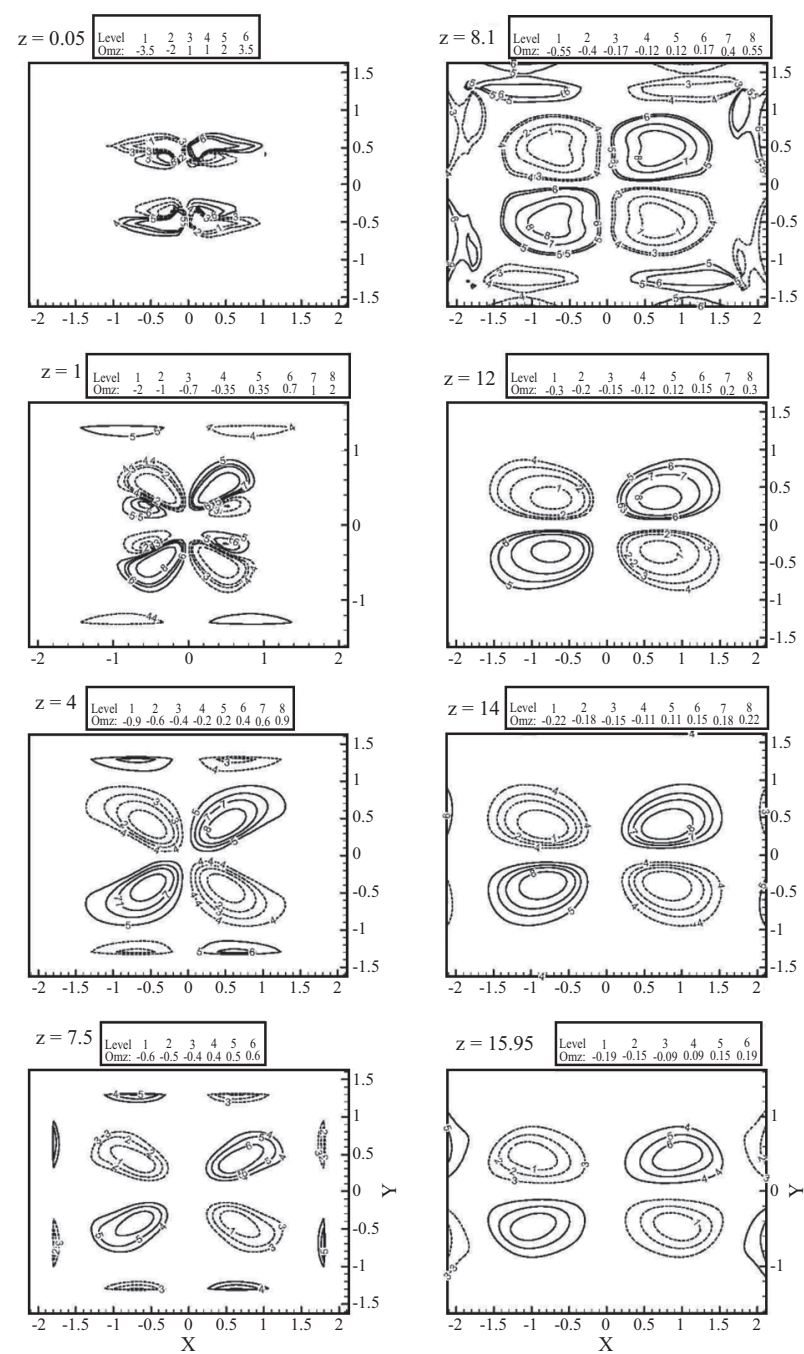

Fig. 7. Contours of streamwise vortices in presence of two rectangular tabs situated at the minor axis ends of the inlet section on $z=0$; $\boldsymbol{R e}=\mathbf{2 0 0}$.

lateral pressure gradients. Following Ref. 5, from every relative high pressure region (Fig. 8) on a channel section we now draw curved arrows embracing each of the neighboring low pressure regions from both sides, and the same procedure is adopted for pressure contours on every streamwise station and for all the investigated flow situations. According to the hypothesis outlined in Ref. 5, the presence of such an arrow in the sectional pressure field (Fig. 8) should ensure the development of a streamwise vortex in the region, and its clockwise/anticlockwise sense will determine if the generated vortex will be of negative/positive strength. Notably, following Ref. 5, our depicted arrows, issuing from the high pressure region and embracing the neighboring low pressure regions, with the pressure contours on different $z=$ constant planes predict growth of streamwise vortices that matches exactly with the corresponding simulated streamwise dynamics, as shown in Fig. 5.

Since developed flow in presence of tabs at the minor axis ends produce a totally different (outflow type) streamwise 

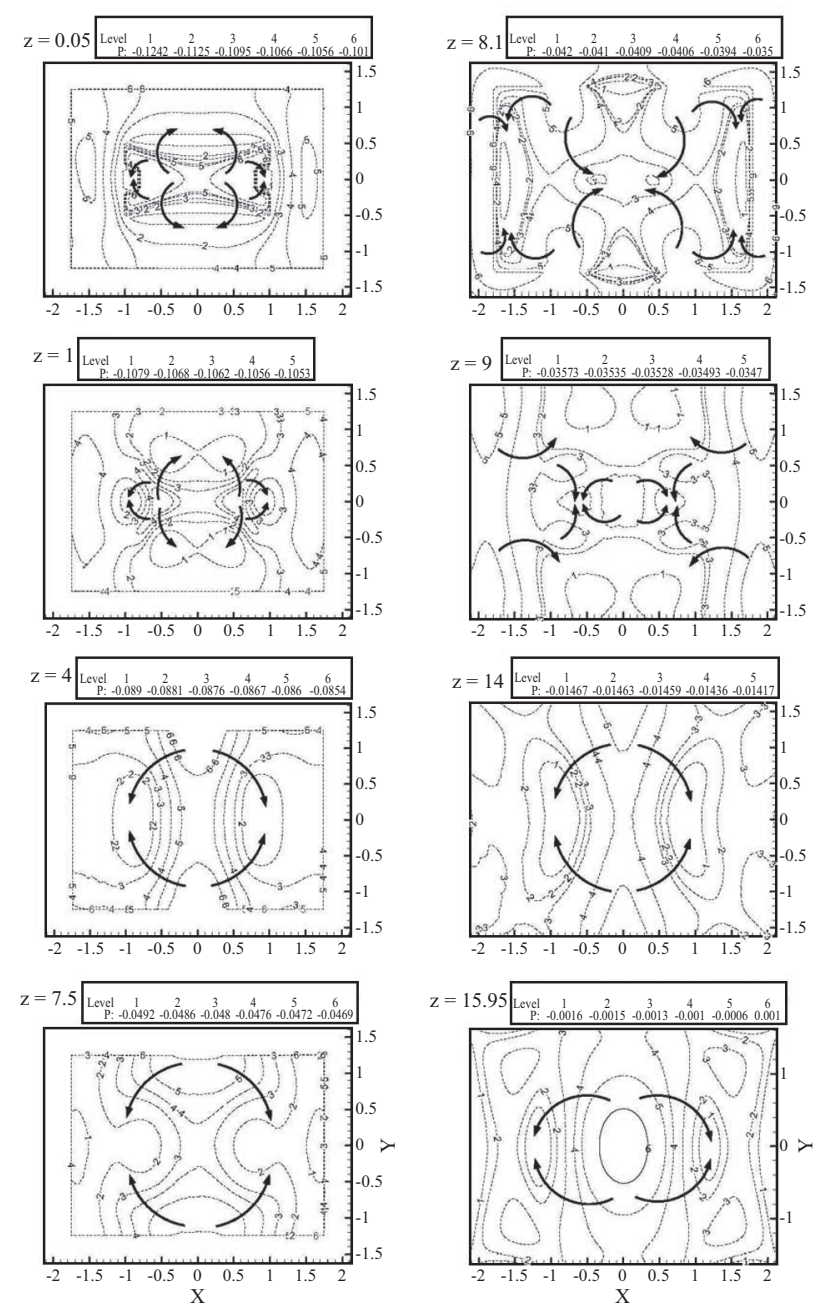

Fig. 8. Contours of pressure $(p)$ in presence of tabs at the major axis ends of the inlet section on $z=0 ; R e=200$.

dynamics (Fig. 7), it is of interest here to examine if a similar pressure analysis can successfully predict growth of such vortices in this flow configuration. Fig. 9 reveals the distributions of iso-contours of $p$ for the flow. The corresponding jet evolution pattern and the associated streamwise dynamics are presented in Figs. 6-7. Notably, the contours (Fig. 9) reveal formation of relative high/low pressure regions on every $z$-plane, and the depicted arrows (Fig. 9) with the pressure contours (issuing from the high pressure regions and embracing existing neighboring low pressure regions) predict local growth of outflow type streamwise vortices on various $z$-planes. Remarkably, the simulated streamwise vorticity distribution (Fig. 7) on the respective planes matches exactly with the predicted local growth/dynamics of the vortices (Fig. 9). Therefore, the presented pressure analysis is seen to efficiently predict the growth of streamwise vortices for the investigated cases.

\section{CONCLUDING REMARKS}

Our simulated results, particularly for flows with tabs, clearly
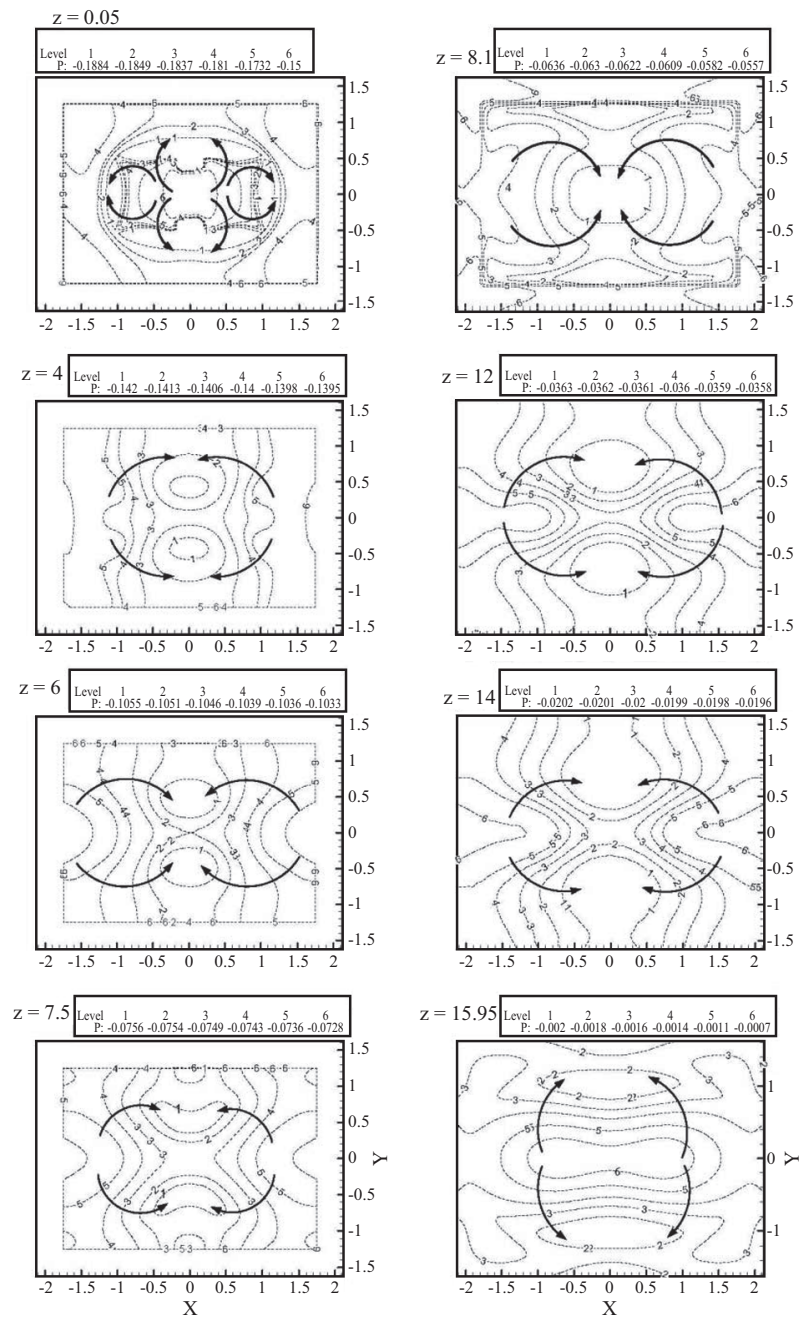

Fig. 9. Contours of pressure $(p)$ in presence of tabs at the minor axis ends; $R e=200$

reveal that the streamwise dynamics of the vortices effectively controls the downstream jet deformation process. With the proper placement of tabs, it has been made possible to allow the growth of a particular type of (favorable) streamwise vortices in the setup. In addition, the presence of the second expansion facilitated generation of additional passive forcing which helped to enhance mixing in the setup through the increased strength of the streamwise vortices. Though inflow type dynamics the streamwise vortices, situated at the ends of the major axis, pushed the jet inward from both sides (forcing it to spread more along the minor axis), and consequently the jet structure became deformed to an another rectangular shape with reversed axial orientation. On the other hand, the placement of the same tiny tabs at the minor axis ends of the inlet channel facilitated the growth of two pairs of outflow type dominant streamwise vortical rollers at the ends of the major axis. Through the outflow type dynamics, the vortical rollers in this case forced the jet to remain spread primarily along the major axis. The pressure contours on different transverse planes reveal formation of several relative high/low pressure 
regions over the flow domain. The non-uniform local flow accelerations from such relative high pressure regions to the neighboring low pressure regions have been noted to induce the generation of the streamwise vortices in the setup.

\section{ACKNOWLEDGMENTS}

Authors gratefully acknowledge many constructive discussions with Prof. Subhasish Dey and Dr. W. C. Yang, and financial support from NSC (grant No. 97-2221-E-001-024), Taiwan.

\section{REFERENCES}

1. Bernal, L. P. and Roshko, A., "Streamwise vortex structures in plane mixing layers," Journal of Fluid Mechanics, Vol. 170, pp. 499-525 (1986).

2. Bradshaw, P., "The turbulent secondary flows," Annual Review of Fluid Mechanics, Vol. 19, pp. 53-74 (1987).

3. Breidenthal, R. E., "Structure in turbulent mixing layers and wakes using a chemical reaction," Journal Fluid Mechanics, Vol. 109, pp. 1-24 (1981).

4. Dhanak, M. R. and De Bernardinis, B., "The evolution of an elliptic vortex ring," Journal of Fluid Mechanics, Vol. 109, pp. 189-216 (1981).

5. Grinstein, F. F., Gutmark, E., and Parr, T., "Near-field dynamics of subsonic free square jets. A computational and experimental study," Physics of Fluids, Vol. 7, pp. 1483-1497 (1995).

6. Hertzberg, J. R. and Ho, C. M., "Time-averaged three-dimensional flow in a rectangular sudden expansion," AIAA Journal, Vol. 30, pp. 2420-2425 (1992).

7. Hertzberg, J. R. and Ho, C. M., "Three-dimensional vortex dynamics in a rectangular sudden expension,” Journal of Fluid Mechanics, Vol. 289, pp. 1-27 (1995).

8. Ho, C. M. and Gutmark, E., "Vortex induction and mass entrainment in a small aspect-ratio elliptic jet," Journal of Fluid Mechanics, Vol. 179, pp. 383-405 (1987).

9. Hussain, F. and Husain, H., "Elliptic jets. Part 1. Characteristics of unexited and excited jets," Journal of Fluids, Vol. 208, pp. 257-320 (1989).

10. Krothapalli, A., Baganoff, D., and Karamcheti, K., "On the mixing of rectangular jet," Journal of Fluid Mechanics, Vol. 107, pp. 201-220 (1981).

11. Liepmann, D. and Gharib, M., "The role of streamwise vorticity in the near-field entrainment of round jets," Journal of Fluid Mechanics, Vol. 245, pp. 643-668 (1992).

12. Sau, A., "Vortex dynamics and mass entrainment in a rectangular channel with a suddenly expended and contracted part," Physics of Fluids, Vol. 14 pp. 3280-3308 (2002).

13. Sau, A., Hwang, R. R., Sheu, T. W., and Yang, W. C., "Interaction of trailing vortices in the wake of a wall-mounted rectangular cylinder," Physical Review E, Vol. 68, 056303 (2003).

14. Sau, A., Sheu, T. W., Hwang, R. R., and Yang, W. C., "Three-dimensional simulation of square jets in cross-flow," Physical Review E, Vol. 69, 066302 (2004).

15. Sau, A., Sheu, T. W., Tsai, S. F., Hwang, R. R., and Chiang, T. P., "Structural development of vertical flows around a square jet in cross-flow," Proceeding of Royal Society London A, Vol. 460, pp. 3339-3368 (2004).

16. Zaman, K. B. M. Q., "Axis switching and spreading of an asymmetric jet: the role of coherent structure dynamics," Journal of Fluid Mechanics, Vol. 316, pp. 1-27 (1996).

17. Zaman, K. B. M. Q. and Raman, G., "Reversal in spreading of a tabbed circular jet under controlled excitation," Physics of Fluids, Vol. 9, pp. 37333741 (1997).

18. Zaman, K. B. M. Q., Reeder, M.F., and Samimy, M., "Control of an axisymmetric jet using vortex generators," Physics of Fluids A, Vol. 6, pp. 778-793 (1994). 